\title{
Program-assisted sizing of a photovoltaic-powered water pumping system
}

\author{
Edward Chikuni \\ Department of Electrical Engineering, Cape Peninsula University of Technology
}

\begin{abstract}
Climate change has had perhaps the most adverse effect on African rural communities where we witness persistent droughts and erratic rain patterns. Peasants often have to walk many kilometres to fetch water of a suspect quality. In these circumstances underground water supplies provide the best hope for them. Often, however, water tables may have receded to such an extent that wells are not a practical proposition. Besides, water is needed not just for domestic use such as food preparation and cleaning, but also for livestock and watering of small gardens. In this paper we present methodology for sizing and designing a photovoltaic pumping system based on components available in Southern Africa. We also show that solar pumping technology has gone past the experimental and prototype stage. Further we strongly put forward the proposition that the technology has clearly matured and in terms of cost, is fast approaching that of choice over other technologies relying on the grid. Currently the region faces an energy shortage and we see not only electricity cuts but those customers that actually are supplied with electricity face increasing and unacceptable tariffs. To make it more convenient we have consolidated the design process in a Visual Basic tool which is easy to use and apply.
\end{abstract}

Keywords: solar energy applications, photovoltaic pumping, water pumping

\section{Introduction}

According to Michael Sawka of the US Army Research Institute of Environmental Medicine, a human being can survive for several weeks without food, but hardly a week without water, even in a cool environment. Water, then, is life and as Hungarian Biochemist and Nobel Laureate Albert
Szent Gyorgyi puts it, 'Water is life's mater and matrix, mother and medium. There is no life without water.'. Yet, we take it for granted as observed many years ago by conservationist Rachel Carson, 'In an age when man has forgotten his origins and is blind even to his most essential needs for survival, water along with other resources has become the victim of his indifference.'

Solar pumping as a technology became available as far back as the late 70's. Each and any of the components used was very expensive and often very suspect in terms of reliability. The systems were also often too complicated for the rural, often backward communities to which they were presented. Several of these were prototypes funded by development agencies. Suppliers were largely in the background and largely passive. The situation now has radically changed and big corporations are now involved. PV panel prices have dropped from US\$300 per Peak watt in the 50's to US\$20 per peak watt in the 70's (Corona technologies, 2010), and the situation now has improved much further. Even figures predicted by economic analysts and reported by Barlow, McNeils and Derrik (1993) of around US\$5 per peak watt are now outdated. Ernst \& Young (2011) in their report predict that for systems above $50 \mathrm{~kW}$, prices will drop to US $\$ 1.00$ per peak watt by 2013 In parts of Africa with good solar radiation such as Namibia and Sudan the viability of Solar PV pumping increased; Eltahir,. AbdelGadir, and Hamad (2009) report radiation levels of up to $7.7 \mathrm{kWh} / \mathrm{m}^{2}$ per day. The situation appears to be progressively and decisively improving and despite distortions introduced by currency fluctuations we see that prices have been dropping, perhaps not as drastically as computers and computer components, but clearly dropping. Figure 1 shows a graphical representation of the trend from the 1950's to the present generation. We see from this that the US\$1.00 per peak watt PV power is a now very realistic prediction. The question now only is, will there be a limit to this price decrease trend? 


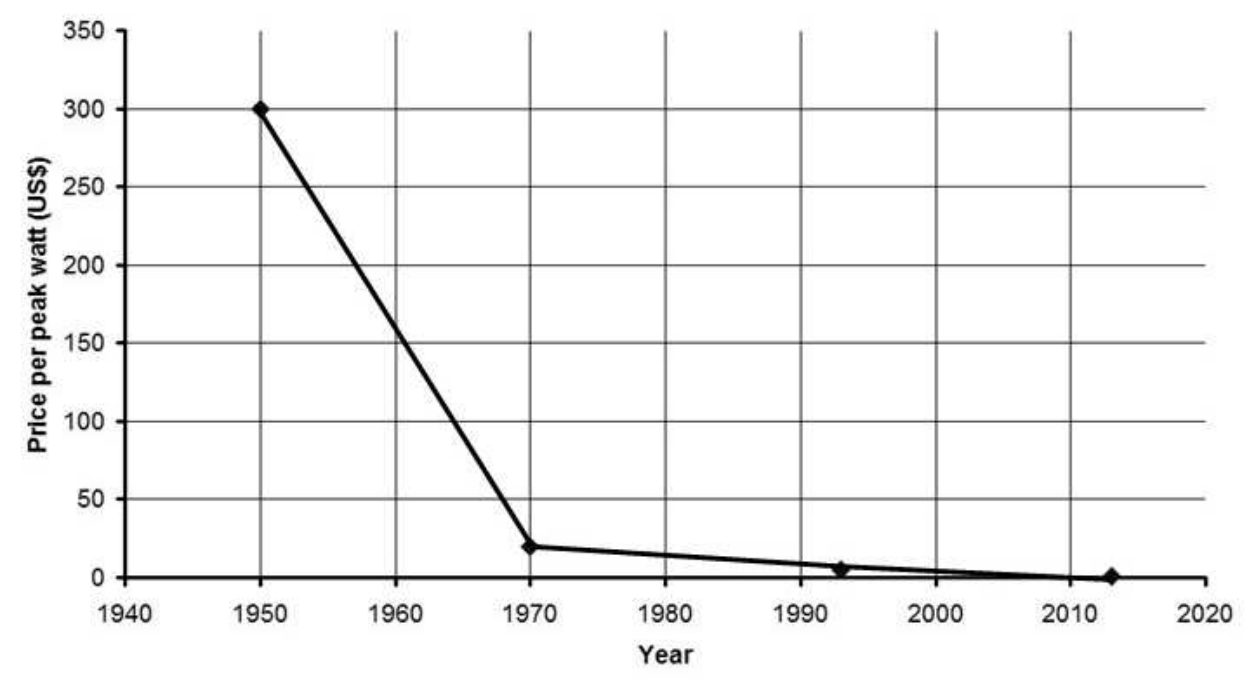

Figure 1: PV price trends

There have also been very positive developments in pump component technologies, especially in electric motors and related controls. Submersible motors have for a long time been favoured for their efficiency. Traditionally they have been single or three phase asynchronous motor types but we see now brushless DC types with new inverter drive systems controlled by Digital Signal Processing hardware and software algorithms.

This paper harnesses and embraces these positive developments and is intended to give a practically implementable design process for a Photovoltaic (PV) pumping system.

\section{The design and sizing process}

Eker (2005) lists several considerations, including information that would be required to be furnished to a service provider of solar pumping systems. Among these is the reliability of the system measured by the loss of load probability (LLP) (Hamidat and Benyoucef, 2009). Assuming that these considerations have been attended to, there are two early considerations in our design methodology:

- Assessment of the solar potential - for this we need to establish the amount of sunshine available in a day, i.e. the peak solar hours (PSH). In most of Southern African and certainly in Namibia we typically have 6 PSHs in a day. For specific sites we can refer to meteorological charts.

- The head in meters - representing the amount of work the pump has to exert in lifting the water from inside the bore-hole or well to the tank. We will see that the head is a function of several factors that includes the roughness of the delivery pipe and its dimensions, (length and diameter). From the above we can have a good idea of the energy requirements of the pump and eventually the pump type and solar array configuration.

\subsection{Pumping energy requirements}

Suppose that on a particular day we wish to determine the energy requirements of a pump. This is determined from:

$$
E=\frac{m g H}{\eta}
$$

$m=$ the mass of the water needed $(\mathrm{kg})$

$g=$ the acceleration due to gravity $\left(\mathrm{m} / \mathrm{s}^{2}\right)$

$H=$ the height from pump to the tank (m)

$\eta=$ Efficiency of the system

With the SI system units as above, E will be in joules. Quite often, however, we need to express the energy in $\mathrm{kWh}(1 \mathrm{kWh}=1$ 'unit' of electrical energy). Then we will use the conversion factor, 1 joule $=2.77778\left(10^{-7}\right) \mathrm{kWh}$

$$
E=\frac{m(9.81)\left(2.77778\left(10^{-7}\right) H\right.}{\eta}
$$

Since we are pumping water (density $1000 \mathrm{~kg} / \mathrm{m}^{3}$ ), we can replace $\mathrm{m}$ by $1000 \mathrm{~V}$ (where $\mathrm{V}$ is the volume of the water in $\mathrm{m}^{3}$ ), thus:

$$
E=\frac{1000 V(9.81)\left(2.77778\left(10^{-7}\right) H\right.}{\eta}
$$

Equation (3) simplifies to the approximate expression:

$$
E=\frac{V H}{367 \eta} \mathrm{kWh}
$$

Equation (4) is quite powerful in that it can quickly tell us how much it will cost to pump the water. If for example, we require 5000 litres of water $(V=5 \mathrm{~m} 3)$ presenting a total head of $50 \mathrm{~m}$ and our pump is $50 \%$ efficient, then $1.36 \mathrm{kWh}$ is required (i.e. $\mathrm{R} 1.36$ ) at $\mathrm{R} 1.00$ per $\mathrm{kWh}$ ). 


\subsection{Calculation of head}

The total head against which the pump must work consists of three main components:

- A dynamic head $\mathbf{H}_{\mathrm{d}}$ (it is known that when pumping action occurs, the water level drops and after several hours stabilises). The level in these stabilised circumstances is called the dynamic head.

- A head due to the elevation of the delivery pipes from the ground, $\mathbf{H}_{e}$

- A head due to friction, $\mathbf{H}_{\mathrm{f}}$

- A velocity head, $\mathbf{H}_{\mathbf{v}}$

The sum of the above is the total head,

$$
\mathbf{H}_{\mathrm{t}}=\mathbf{H}_{\mathrm{d}}+\mathrm{H}_{\mathrm{e}}+\mathbf{H}_{\mathrm{f}}+\mathbf{H}_{\mathrm{v}}
$$

Figure 2 shows the general arrangement.

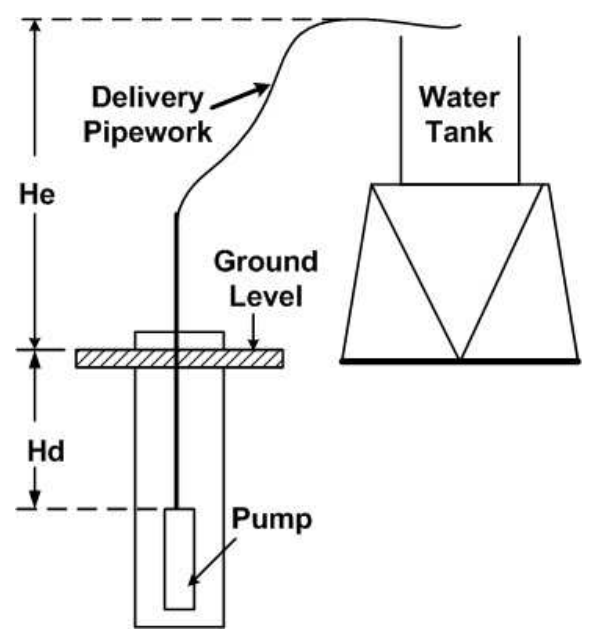

Figure 2: An elementary pumping system

In Equation (5), $\mathrm{H}_{\mathrm{d}}, \mathrm{H}_{e}$ and $\mathrm{Hv}$ can be readily calculated or measured. The friction head is somewhat problematic.

\subsubsection{Calculation of the friction head}

The Darcy Equation is a fluid dynamics equation that attempts to predict energy loss during fluid flows in pipes taking into consideration velocity of flow and frictional resistance. For turbulent flow through a circular pipe the following flow is used:

$$
H_{f}=\frac{4 f l}{d} \frac{c^{2}}{2 g}
$$

Where

$\mathrm{f}=$ friction factor

1 = length of pipe

$\mathrm{d}=$ pipe diameter

$\mathrm{c}=$ mean velocity of fluid

Since the rest of the variables in Equation [6] can readily be determined, it is the friction factor, $\mathrm{f}$, that is more problematic. For smooth flow and smooth pipes, Blasius equation is applicable:

$$
f=\frac{0.079}{\operatorname{Re}^{1 / 4}}
$$

Where Re is Reynolds number. If Re is less than 2000, Equation [7] can further be simplified [6] to:

$$
f=\frac{64}{\mathrm{Re}}
$$

If however Re is significantly higher than 2000 , a graphical technique which involves the use of the Moody Chart and the Darcy equation is used. The calculation example which follows illustrates these concepts.

\section{Head calculation example}

The requirement is for 5000 litres per day. We shall designate this $Q_{\text {day }}$. If the pump was able to work 24 hours per day, then we would simply convert this figure to $\mathrm{m}^{3} / \mathrm{s}$. To convert litres per day to cubic meters per second we use the conversion factor, $1.15741\left(10^{-8}\right)$. So that $5000 \mathrm{l} / \mathrm{day}=5000(1.15741)$ $\left(\left(10^{-8}\right) \mathrm{m}^{3} / \mathrm{s}\right.$. We also need to know the number of peak sunshine hours (PSH) available and this we can obtain from weather data. Suppose the PSH is 6 , then the real pump capacity should be:

$$
Q_{\text {pump }}=\frac{24}{P S H}(1.15741)\left(10^{-8}\right)\left(Q_{\text {day }}\right)
$$

For $\mathrm{Q}_{\text {day }}=5000$ litres/day, and $\mathrm{PSH}=6, Q_{\text {pump }}=$ $0.000231 \mathrm{~m}^{3} / \mathrm{s}$

Often pump flow rate is expressed in litres per minute and if this is needed we simply multiply by 60000 , thus: $Q_{\text {pump }}=(0.000231)(60000)=13.86$ litres per minute.

\subsection{Friction and velocity head}

The velocity head is given by:

$$
H_{v}=\frac{v^{2}}{2 g}
$$

Where $v=\frac{Q_{\text {pump }}}{A}$

Where $\mathrm{A}$ is the pipe inside section area, $=\frac{\pi}{4} d^{2}$

Suppose that the pipe diameter is $25 \mathrm{~mm}$, then $\mathrm{A}=$ $0.000491 \mathrm{~m}^{2}$. Then from equation (10), $\mathrm{V}=$ $(0.000231 / 0.000491)=0.4716 \mathrm{~m} / \mathrm{s}$

\subsubsection{Friction head calculation: important decision rule}

From Renault's equation:

$$
\operatorname{Re}=\frac{\rho v d}{\mu}
$$

Where, $\rho=$ fluid density $\left(\mathrm{kg} / \mathrm{m}^{3}\right)$

$v=$ velocity $(\mathrm{m} / \mathrm{s})$

where, $d=$ pipe diameter $(m)$

$\mu=$ dynamic viscosity 
This allows us determine the roughness of the flow, that is, whether it is laminar (smooth) or rough (turbulent). If it is laminar we can use Equation [8] to determine the friction factor required for equation 6. If turbulent, we need to employ a graphical approach.

Upon substituting given values into Equation [11]:

$$
\operatorname{Re}=\frac{(1000)(0.4716)(0.025)}{(0.001)}
$$

The decision rule (Higher Academic Education Centre, 2010) is that if $\mathrm{Re}$ is 2000 or less, we use equation (8), if much more we use a graphical method e.g. Moody Chart (Eker, 2005). In this example, the flow is clearly turbulent and the graphical approach would be appropriate. Since a Moody Chart can be unwieldy, several online friction factor calculators are now available. One calculator is by LMNO Engineering and Research Software limited (2010). For the determination of $\mathrm{Re}$, the kinematic rather than dynamic viscosity is used. There is a relation but this is exactly not linear as shown by Table 1. This table shows that Dynamic and Kinematic viscosities are both dependent on temperature. Their actual physical relations are:

$$
\text { Kinematic viscosity }=\frac{\text { Dynamic viscosity }}{\text { Fluid density }}
$$

Table 1: Kinematic and dynamic viscosity of water (SI units)

Source: Engineering Toolbox.com

\begin{tabular}{lcc}
\hline Temp $\left({ }^{\circ} \mathrm{C}\right)$ & $\begin{array}{c}\text { Dynamic } \\
\left(\mathrm{Ns} / \mathrm{m}^{2}\right) \times 10^{-3}\end{array}$ & $\begin{array}{c}\text { Kinematic } \\
\left.\mathrm{m}^{2} / \mathrm{s}\right) \times 10^{-6}\end{array}$ \\
\hline 0 & 1.787 & 1.787 \\
\hline 5 & 1.519 & 1.519 \\
\hline 10 & 1.307 & 1.307 \\
\hline 30 & 1.002 & 1.004 \\
\hline 40 & 0.798 & 0.801 \\
\hline 50 & 0.653 & 0.658 \\
\hline 60 & 0.547 & 0.553 \\
\hline 70 & 0.467 & 0.475 \\
\hline 80 & 0.404 & 0.413 \\
\hline 90 & 0.355 & 0.365 \\
\hline 100 & 0.315 & 0.326 \\
\hline
\end{tabular}

The dependence of density on fluid temperature is the reason for relatively small differences at elevated temperatures. For water temperatures under $30^{\circ} \mathrm{C}$, we can arrive at kinematic velocity by dividing dynamic viscosity by 1000 . The LMNO Calculator requires some knowledge of the viscosity of the water as well as a numerical value for the roughness of the pipe. The equations used for the calculations are:

$$
\begin{aligned}
& \text { for } \operatorname{Re} \leq 2100 f=\frac{64}{\operatorname{Re}} \text { where } \operatorname{Re}=\frac{\rho v d}{\mu} \\
& \text { for } 5000 \leq \operatorname{Re} \leq 10^{8} \text { and } 10^{-6} \leq \frac{e}{d} \leq 10^{-2},
\end{aligned}
$$

Where $e$ is the roughness of the pipe:

$$
\text { then } f=\frac{1.325}{\left[\ln \left(\frac{e}{3.7 d}+\frac{5.74}{\operatorname{Re}^{0.9}}\right)\right]^{2}}
$$

Another calculator, that of Pneucon Valley Springs, California USA (2010), is an approximate approach based on the most common operating environments and yields good results for water without requiring knowledge of viscosities and requesting only the type and qualitative description of the roughness and type of pipe. The results obtained for this example using the two programs are not too far apart, ( $\mathrm{f}=0.0366$ for LMNO (using pipe diameter of $0.025 \mathrm{~m}$, roughness 0.00007 , dynamic viscosity of $1.14 \times 10^{-3} \mathrm{Ns} / \mathrm{m}^{2}$ ) and $\mathrm{f}=$ 0.0295 for Pneucon). The LMNO results would be more accurate since exact pipe fluid parameters are used. For the example we shall use the LMNO 0.0366, so that from Equation (6),

$$
\begin{aligned}
H_{f} & =\frac{4 f l}{d} \frac{c^{2}}{2 g} \\
H_{f} & =\frac{4(0.0366)(50)}{0.025} \frac{(0.4716)^{2}}{2(9.81)} \\
& =3.31 \mathrm{~m}
\end{aligned}
$$

\subsubsection{Velocity head}

The velocity head calculation is quite straight forward and is given by equation (9). On substitution for this example, we find $\mathrm{H}_{\mathrm{v}}=0.011 \mathrm{~m}$. This is quite small for these operating conditions.

\subsubsection{Total head}

The total head is given by equation (5). Assuming a dynamic head of $30 \mathrm{~m}$, a delivery head of $5 \mathrm{~m}$, then the total head $\mathbf{H}_{\mathbf{t}}$ is $(30+5+3.31+0.011)=38.3 \mathrm{~m}$.

\section{Determination of power requirements}

In Equation (4) if we divide the $\mathrm{kW}$ hours by hours, we get $\mathrm{kW}$ required by the pumping system, thus:

$$
\begin{aligned}
& \frac{E}{h r}=\frac{\frac{V}{h r} H}{367 \eta} \\
& P_{(k W)}=\frac{Q_{p h} H}{367 \eta}
\end{aligned}
$$

Where $Q_{p h}$ is flow in $\mathrm{m}^{3} / \mathrm{h}$.

To convert $Q_{\text {pump }}$ into $Q_{\text {ph }}$ we multiply by 3600 . On substitution we have an assuming $30 \%$ pump efficiency. This value depends on the pumping 
technology used and Protogeropoulos and Pearce [11] cite values between $30 \%$ and $50 \%$.

$$
\begin{aligned}
P_{(k W)} & =\frac{(0.833)(38.33)}{367(0.3)} \\
& =0.290 \mathrm{~kW}
\end{aligned}
$$

\section{Selection of PV array}

The size of PV array has to have a relationship to the pump requirements. However, we need to acknowledge the depreciation of the panel due to ageing and environmental features (dust, etc). If a depreciation of 20 percent is allowed for:

$$
\begin{aligned}
& P_{\text {(Array) }}=P_{(\mathrm{kW})}(1.2) \\
& =(0.290)(1.2 \\
& =0.384 \mathrm{~kW}
\end{aligned}
$$

\section{A $350 \mathrm{~W}$ rated array would be suitable.}

\section{Selection of the pump}

There are several approaches and philosophies in the pump selection, for example, by Blankson and Baker (1994), ITDG or Protogeropoulus and Pearce (2000). In all these, efficiency, cost and maintainability are important criteria. Our illustrative graph (Figure 3) uses US units so some conversions need to be done. The flow rate is approximately 13.8 litres per minutes, i.e. about 3.7 US gallons per minute. The head is $38.3 \mathrm{~m}$ (125ft), and using Figure 3, we find that for the Grundfos $6 \mathrm{SQF}-2$ pump (Grundfos, 2012) and applying the near $130 \mathrm{ft}$ curve, the pumping requirement will be about
$240 \mathrm{~W}$. This figure is comfortably within the figure calculated in section 4 .

\subsection{Pump motors}

In general submersible pumps are more efficient than above ground types. Traditional DC motors e.g. the DC Series motor (Kathib, Mohamed, Nowshad, 2009) are now outdated for any drive application, and we now have the brushless DC motors which will need an inverter or alternatively special squirrel motors with advanced controllers (e.g. field oriented control) which 'mimic' the DC machine's excellent drive characteristics (speedtorque).

\section{Utilisation of Visual BASIC (VB) tool}

To avoid the tedious calculation procedure outlined, a Visual Basic tool was designed that automates the process. This also allows rapid evaluation of pumping solutions under varying scenarios. This tool is PVPDESYS.

\subsection{Data input}

PVP_SYSD requires the following data:

- Daily water requirement in litres (V)

- Peak solar hours (PSH)

- Pipe diameter (d)

- Pipe roughness $(e)$

- Dynamic viscosity

- $\quad$ Dynamic head (Hd)

- $\quad$ Elevation: ground to delivery point $(\mathrm{He})$

- Pipe length (L)

Figure 4 shows the input-output screen.

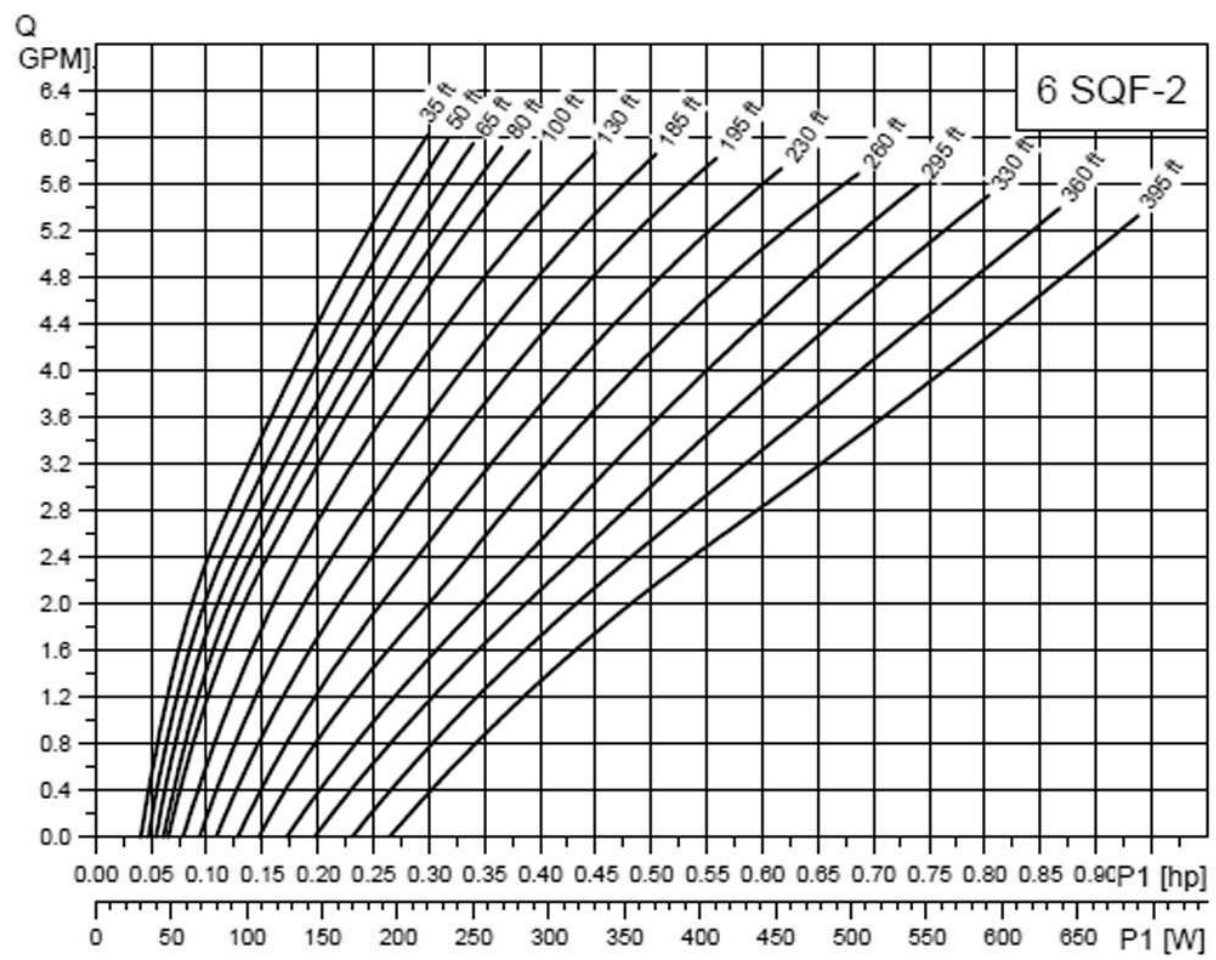

Figure 3: A pump selection chart 


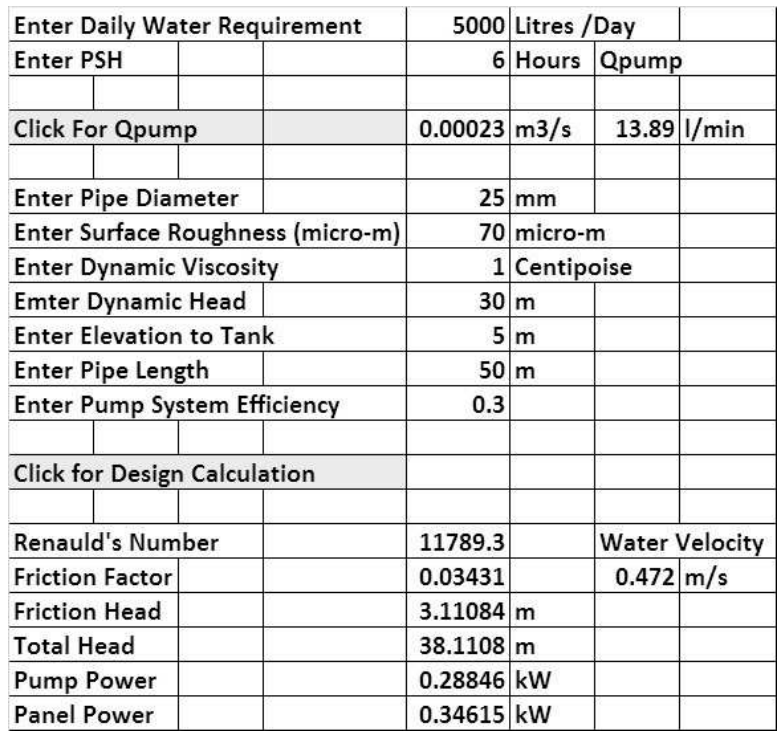

Figure 4: PVPDESYS

\section{Consideration of other factors}

Moussi, Saadi, Betka \& Asher (2003) describe to a good depth PV systems and the practical issues involved. For the solar panel itself, depreciation has been also been discussed. Clearly other factors can become relevant, which we discuss.

\subsection{Solar panel: Maximum power point tracking}

To obtain optimum power out of a solar panel during varying weather (e.g. low irradiation) and load conditions, controllers called maximum power point trackers are used, beginning from the simpler designs - to more complex fuzzy logic or chaos theory based algorithms (Kathib et al., 2009), (Koner et al., 1992), (Lim et al., 2000), (Zhou et al., 2011). It needs to be pointed out that elaborate complex control systems could affect not only system cost, but overall maintainability which ought to be considered before implementation. In any case, for sunny regions, some of the reasons for requiring complex MPPT control systems, such as low irradiation may not apply.

\subsection{Solar panel: Orientation and control}

Manufacturers give PV performance data based usually under standard test conditions (STC) To obtain maximum performance of out of a solar panel, there are a number of other factors that need to be considered. Among these are:

- Wind direction

- Wind speed

- Humidity

- Azimuth and tilt angle

In the end all these have the effect on the power output of the panel. When these need to be considered a single control algorithm is not enough. With adequate sensing of output voltage and current, the
MPPT controller can help boost the performance, by boosting, as necessary, converter voltage and therefore battery current. A separate controller will, if economically justified, be needed to adjust the tilt angle. In fact, a study at UCT by Bekker (2007), shows that such adjustments have little positive influence on the panels power output. Some trackers are used in less PV favourable locations of Europe, even then for higher panel ratings exceeding hundreds of kilowatts. More common are seasonal adjustments of tilt angle, but even in these cases performance enhancement is only of the order of 3-5\% (Liu, 2010).

\section{Conclusions}

In this paper we have expounded the increasing importance of solar energy and the vital role in our world which is becoming increasingly intolerant to carbon. Photovoltaic pumping is a particularly attractive solution for the provisions of water for both humans and livestock for drier parts of Africa such as Namibia and Botswana. As we have seen the design process has many steps and the formulas though not very complex require certain care to avoid errors. In this regard, computerisation of the process is a step forward. We have found PVS_SYSD to be quite useful for overall system design especially in cases where many scenarios have to be considered. The scope of our investigation has not allowed us to delve into real, quite profound technological issues evolve such as the quest for optimum efficiency of the array through maximum power point tracking, temperature compensation (Moussi, Saadi, Betka \&Asher, 2003), or indeed economic issues, such as life cycle costing (Eltahir, AbdelGadir, Mahmoud \&. Hammad, 2011).

\section{References}

Bankston, Jr., J. D. and Baker F.E.. 1994. Selecting the Proper Pump. Southern Regional Aqua-culture Centre (SRAC), publication 372, December, 1994.

Barlow, R., McNelis B. and Derrick A. (1993). 'Solar Pumping', Intermediate Technology Publications, ISBN 1853391794

Bekker, B. 2007. Irradiation and PV array energy output, cost, and optimal positioning estimation for South Africa. Journal of Energy in Southern Africa 18 (2): $16-25$.

Coronatech. 2010. Overview of solar power. http://coronatech.org/overview_of_solar_power.htm, Accessed: November 6, 2010.

Eker, B. 2005. Solar powered water pumping systems. Trakia Journal of Sciences 3 (7): 7-11.

Eltahir K. AbdelGadir M. and Mahmoud A. 2011. http:// gcreader2008.ju.edu.jo/PDF/501-c.pdf Accessed: February 13, 2011.

Grundfos. 2012. SQ flex renewable energy based water supply systems, $50 / 60 \mathrm{~Hz}$. http://ca.grundfos.com/content/dam/GCA/Literature/Product\%20Guide/LSPTL0 
14_1211_SQFlex.pdf. Accessed 13 February, 2012.

Hamidat, A. and Benyoucef B. 2009. Systematic procedures for sizing photovoltaic pumping system, using water tank storage. Energy Policy 37: 1489-1501.

Higher Education Academy Education Centre 2010. www.engsc.ac.uk/.../Headlossduetofrictionincircularpipes.doc. Accessed: November, 2010.

ITDG, Schumacher Centre for Technology and Development. 2011. Solar (PV) water pumping. www. cedecap.org.pe/uploads/biblioteca/109bib_arch.pdf. Accessed February 12, 2011.

Kathib, T.T.N, Mohamed, A. \& Nowshad, N. 2009. A new controller scheme for photovoltaics power generation systems. European Journal of Scientific Research 33 (3): 515-524.

Koner, P.K.J., Joshi, J.C. \& Chopra, K.L. 1992. Matching analysis of photovoltaic powered DC series motors and centrifugal pumps by varing motor constants. International Journal of Energy Research 16 (4): 301-313.

Lim Y.H. and Hamill, D.C. 2000. Simple maximum power point tracker for photovoltaic arrays. Electronics Letters 36 (11).

LMNO Engineering, Research, and Software, Ltd. 2010. www. lmnoeng.com/moody.htm, Accessed November 6, 2010.

Moussi, A. Saadi, A., Betka, E.T. and Asher M.G. 2003. Photovoltaic pumping systems, technologies, trends. Larhyss Journal, 2 June 2003: 127-150.

Pneucon Process Technologies. 2010. www.pneucon.co. $\mathrm{kr} /$ Techinform/vmd03.htm, Acessed, November 6, 2010.

Protogeropoulus, G., and Pearce, S. 2000. Laboratory evaluation and system sizing charts for a 'second generation' direct PV powered low cost submersible pump. Solar Energy 68 (5): 453-474.

Runsheng T., and Liu X. 2010. Installation design of solar panels with seasonal adjustment of tilt-angles. Power and Energy Engineering Conference (APPEEC), 2010 Asia-Pacific.

Zhou, L. Chen Y., Guo K., and Jia, F. 2011. New approach for MPPT control of photovoltaic system with mutative-scale dual-carrier chaotic search. IEEE Transations on Power Electronics 6 (4).

Received 1 March 2011; revised 29 August 2011 